\title{
Hair Cortisol and Perceived Stress in Health-studies Students during Summer Break and Fall Term
}

\author{
Mezentsef $\mathrm{A}^{1}$, Jackson $\mathrm{P}^{1}$, Baig $\mathrm{U}^{1}$, Fairclough $\mathrm{JL}^{1}$, Brooks $\mathrm{C}^{2}$ and Mitroka $\mathrm{J}^{*}$ \\ ${ }^{1}$ Gregory School of Pharmacy, Palm Beach Atlantic University, West Palm Beach, FL, USA \\ ${ }^{2}$ School of Nursing, Palm Beach Atlantic University, West Palm Beach, FL, USA
}

\begin{abstract}
The objective of this study was to evaluate stress in health-studies students over time periods when classes are not in session (Summer) and are in session (Fall) using both a survey instrument and hair cortisol levels. Cortisol is produced by the adrenal cortex in response to stress. Chronic elevations in cortisol are associated with adverse effects on the cardiovascular, musculoskeletal, and central nervous system. As such, cortisol levels can serve as a biomarker for stress. Hair is a particularly useful matrix for measuring cortisol in the body since it grows at a uniform rate, about $1 \mathrm{~cm} / \mathrm{month}$, and thereby provides a time-averaged index of exposure to cortisol. A small bundle of hair (about 50 strands) was collected as close to the scalp as possible from Nursing and Pharmacy students at the beginning of the Fall semester (representing hair grown over the Summer when no classes were in session) and again at the end of Fall semester (representing hair grown during the semester, when classes were in session). A three $\mathrm{cm}$-portion of each hair bundle was cut from the scalp end, ground, extracted, and analyzed by LC/MS/MS. The students also completed a survey to provide subjective feedback about perceived stress levels. These surveys were completed at the time of the hair collections. The analytical results showed no differences in hair cortisol concentrations between Summer and Fall or between Pharmacy and Nursing students. Perceived stress levels; however, were higher in Fall than Summer and somewhat higher in pharmacy students than nursing students during the Fall. The results of this study suggest that health-studies students experience psychological stress during the time classes are in session, but this perceived stress may not result in concomitant elevations in cortisol.
\end{abstract}

Keywords: Hair cortisol levels; Psychological stress; Fall term

\section{Background}

Stress is a common feature of higher education and, particularly, professional education and training. While mild to moderate intermittent stress-such as occurs prior to an exam-can be beneficial, prolonged stress can have adverse emotional, academic, and health consequences.

Evaluation of psychological stress in health science students is abundant, based primarily of self-perceived stress using survey instruments [1-3], but relatively few examples of physiological stress, using objective biomarkers, have been published [4-7]. The purpose of the present study was to evaluate stress in pharmacy and nursing students using both a measure of psychological stress and a measure of physiological stress. Psychological stress was evaluated using the Perceived Stress Scale (PSS), an instrument which has established validity and reliability [8]. Physiological stress was evaluated by determining hair-cortisol concentrations, a relatively new technique which has been used in a wide variety of situations as a biomarker of long term stress [9-11]. The study was approved by the Palm Beach Atlantic (PBA) University Institutional Review Board (IRB) which has jurisdiction over both the Gregory school of Pharmacy and the School of Nursing.

Cortisol is a well-established biomarker for physiological stress [12] or what has been called allostatic load [13,14]. Typically, samples of blood, urine, or feces are the matrices analyzed to estimate systemic exposure to the hormone. The major problem with these matrices is that they only provide a "snapshot" in time of actual cortisol concentrations and the associated degree of stress. Recently, the use of hair as a matrix has been shown to be of value for assessing long term time-averaged systemic exposure to cortisol [9]. Cortisol appears to be incorporated into the hair via passive diffusion from blood, and studies in animals indicate that hair cortisol concentrations correlate well with urinary cortisol concentrations [15]. Human hair grows at approximately $1 \mathrm{~cm} / \mathrm{month}$ and the cortisol trapped in the hair shaft appears to be well retained over a 3 to 6 month period of growth [16]. As such, hair provides, at least in principle, an ideal matrix for assessing long term concentrations of cortisol in the body. Although there are several techniques for analyzing hair samples for cortisol, the "gold standard" method is extraction with methanol followed by LC/MS/MS analysis of the extracts [9].

Our aim in the present study was to evaluate the use of hair cortisol concentrations as an objective biomarker of stress in students. In brief, cohorts of volunteer nursing and pharmacy students were evaluated for stress experienced during the Summer before Fall term (when no classes were in session) and during the Fall term of school (when classes were in session). Pharmacy students in their first two years of professional study only were included in the study to best age-match the Nursing students.

\section{Materials and Methods}

\section{Hair collections}

Hair was collected from student volunteers of both sexes during

*Corresponding author: Mitroka J, Gregory School of Pharmacy, Palm Beach Atlantic University, West Palm Beach, FL, USA, Tel: +5618032725; Fax: +5618032703; E-mail: james_mitroka@pba.edu

Received December 19, 2016; Accepted January 16, 2017; Published January 23, 2017

Citation: Mezentsef A, Jackson P, Baig U, Fairclough JL, Brooks C, et al. (2017) Hair Cortisol and Perceived Stress in Health-studies Students during Summer Break and Fall Term. J Steroids Horm Sci 8: 183. doi: 10.4172/2157-7536.1000183

Copyright: (c) 2017 Mezentsef A, et al. This is an open-access article distributed under the terms of the Creative Commons Attribution License, which permits unrestricted use, distribution, and reproduction in any medium, provided the original author and source are credited. 
the first week of the Fall term and again during the last week of the Fall term. Hair was collected from 37 pharmacy and 33 nursing students ranging in age from 20 to 37 years old. As such, each student served as his or her own control. No provision was made for the possibility of seasonal variation in cortisol. Samples of hair were taken from the posterior apex (crown) of the head as close as possible to the scalp with the aid of a thin comb placed against the skin. No exclusions were made for hair that was treated with hair dye or bleach. A section of hair was separated across about three $\mathrm{cm}$ of the face of the comb and cut such that the total sample of hair as a bundle (about 50 strands) was about the diameter of a pencil. The hair was placed in a piece of aluminum foil and stored at room temperature for later processing. Three-cm sections of hair, represented about three months of hair growth, were cut from the scalp end of each hair bundle for analysis. Although students initially experienced some trepidation about the cosmetic effect of the procedure, loss of this small amount of hair was undetectable.

\section{Hair cortisol analysis}

Concentrations of cortisol in hair were determined by a modification of the method of described by Chen and coworkers [17]. Each hair sample was washed to remove excess oils by vortex mixing with $4 \mathrm{~mL}$ of dichloromethane three times. The washed samples were dried under a stream of nitrogen at room temperature and ground to a powder using a Wig-L-bug $^{\text {Tix }}$ mixer. About $50 \mathrm{mg}$ of the hair powder, accurately weighed, was transferred to a $15 \mathrm{~mL}$ plastic centrifuge tube along with $1.5 \mathrm{~mL}$ of methanol. The tubes were vortexed briefly and placed on a mechanical shaker overnight to extract the cortisol. The tubes were centrifuged, the methanolic extract was transferred to a second set of tubes, and the hair residues were each reextraced twice by vortexing with an additional 0.5
$\mathrm{mL}$ of methanol. The methanolic extracts for each hair sample were combined and spiked with $2.66 \mathrm{ng}$ of $\mathrm{d}_{4}$-cortisol (internal standard) in $0.2 \mathrm{ml}$ of methanol. The spiked extracts were evaporated under a stream on nitrogen at $37 \mathrm{deg}$. The dried residues were reconstituted in $0.3 \mathrm{ml}$ of 50:50 methanol water, $0.1 \%$ formic acid. The reconstituted samples were passed through a 0.2 micron nylon filter and $0.05 \mathrm{ml}$ of the filtrate was injected into the LC/MS/MS, in duplicate, for analysis of cortisol. Cortisol was detected using multiple-reaction monitoring (MRM) in the negative-ion electrospray (ESI) mode. Details of the analytical procedure are shown in Appendix 1.

\section{Perceived Stress evaluation}

Perceived stress was evaluated using a validated survey instrument, namely the Perceived Stress Scale (PSS-10). The PSS-10 is a ten-item questionnaire that asks respondents to rank their stress perceptions and experiences in the preceding month on a scale of 0 (never) to 4 (very often). Perceived stress was evaluated at the time of the hair sample collections at the beginning and end of the Fall term. Students were asked to reflect on their overall level of stress for the previous three months in order to obtain results over the same time interval evaluated for hair cortisol. Total (summated) scores for the 10 question inventory were used to characterize perceived stress (Appendix 2).

\section{Statistical analysis}

Preliminary and primary analyses were conducted using IBM SPSS v.22 statistical software. Preliminary analyses were used to explore the data and to determine whether it was appropriate to employ parametric or non-parametric techniques. Primary analyses consisted of a Wilcoxon Signed-Rank Test to determine if there were significant

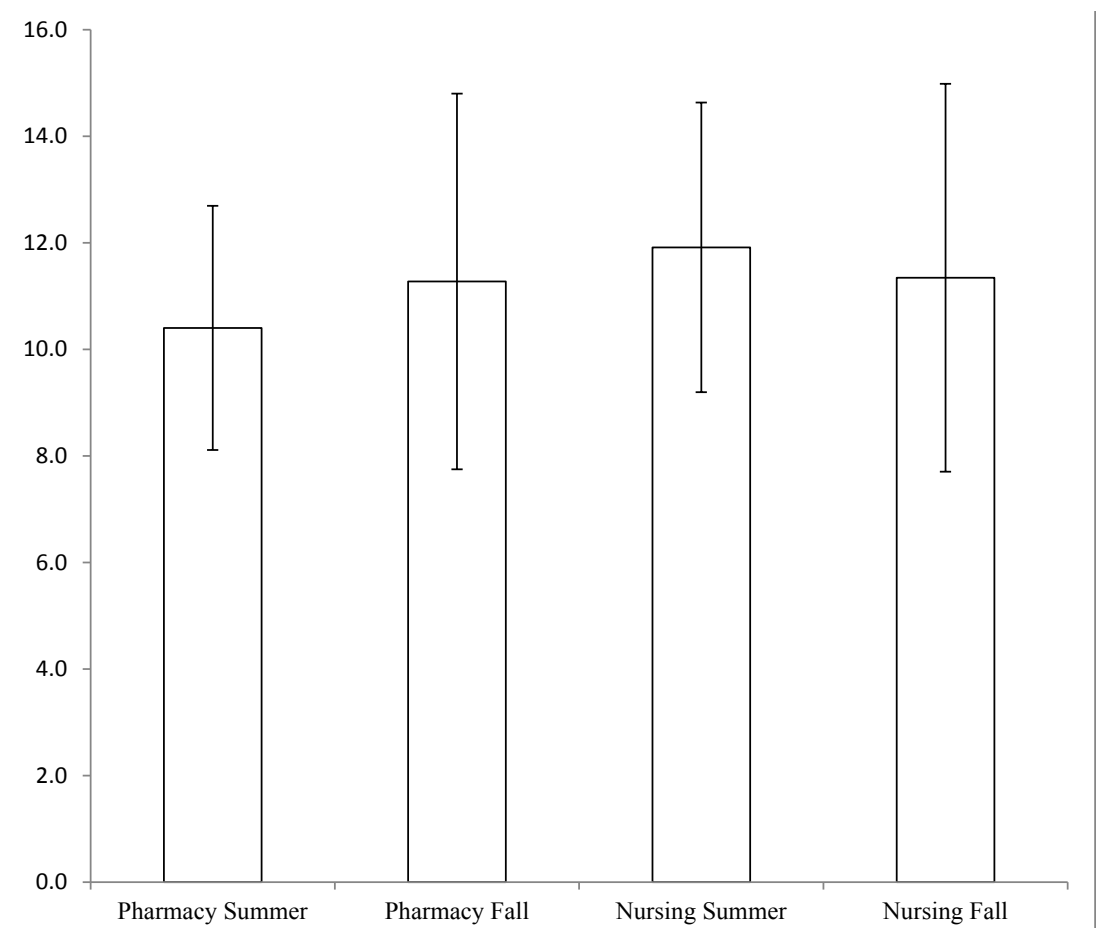

Figure 1: Concentrations of cortisol in hair (pg/mg) of pharmacy and nursing students (Mean $\pm 95 \%$ confidence intervals). Three-cm portions of hair were collected from students at the end of both Summer break and Fall term. The samples were extracted with methanol and the extracts were analyzed for cortisol by LC/MS/ MS. No significant differences $(p<0.05)$ were found among the groups. 
differences in students' cortisol concentrations and perceptions of stress over time; an independent samples Mann-Whitney $U$ test was used to determine if there were significant differences between pharmacy and nursing students' perceptions of stress and cortisol concentrations at baseline (Summer) and/or the end of the Fall semester.

\section{Results}

Concentrations of cortisol in hair varied over 50 fold, from a low value of 2 to a high value of $117 \mathrm{pg} / \mathrm{mg}$. Mean concentrations were similar, at about $10 \mathrm{pg} / \mathrm{mg}$, for all four groups, as shown in Figure 1. No significant differences were observed between nursing and pharmacy students for Summer and Fall term sample periods. Pairwise comparison of concentrations of cortisol hair increased in some students and decreased in other students between Summer and Fall with no clear trend in either direction (Figure 2).

Mean values for perceived stress ranged from 14 to 21 for the four subject groups. Perceived stress levels were higher for Fall vs. Summer for both pharmacy and nursing students as shown in Figure $3(\mathrm{p}<0.01)$. Perceived stress levels were higher for Pharmacy compared to Nursing students during the Fall $(\mathrm{p}<0.05)$.
Values for hair cortisol concentrations varied in an independent manner from perceived stress. Linear regression analysis showed a correlation coefficient of near zero for the two factors as shown in Figure 4.

\section{Conclusion and Discussion}

Based on the results of this study, both the pharmacy and the nursing students at Palm Beach Atlantic University experienced greater perceived stress when classes were in session (during Fall) than when classes were not in session (during Summer). Also, the perceived level of stress during classes was somewhat higher in pharmacy compared to nursing students. The changes in perceived stress; however, were not accompanied by corresponding changes in hair cortisol concentrations.

Our results showing increased perceived stress in pharmacy students while classes are in session are consistent with the results reported by Hirsch [18] and Marshall et al. [19] using survey instruments. Neither of these studies, however, utilized any objective measure of stress.

Only a few studies have evaluated the relationship between hair cortisol concentrations and stress in students. A study conducted in

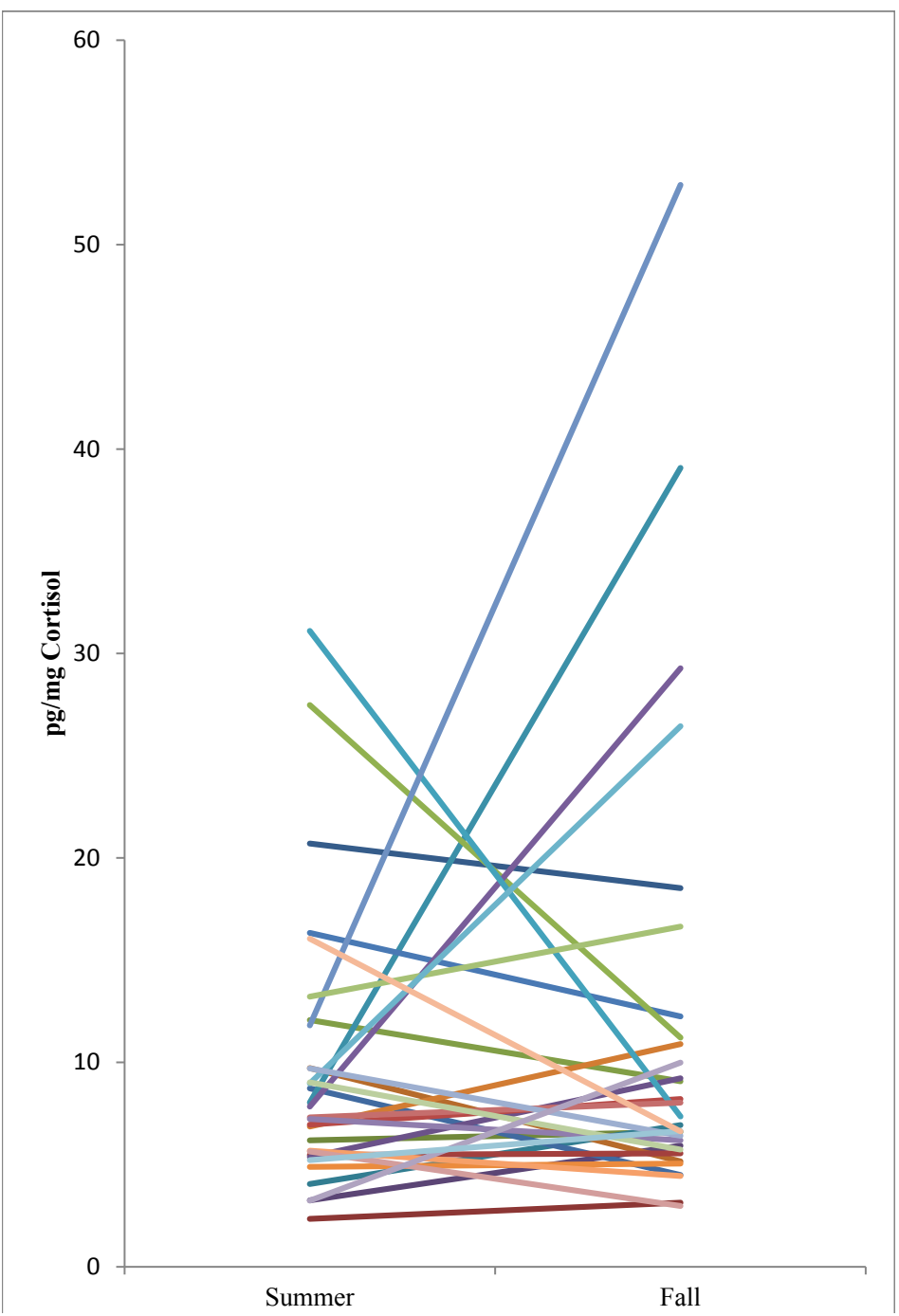

Figure 2: Pairwise comparison of hair-cortisol concentrations for individual pharmacy students between two collection periods. 


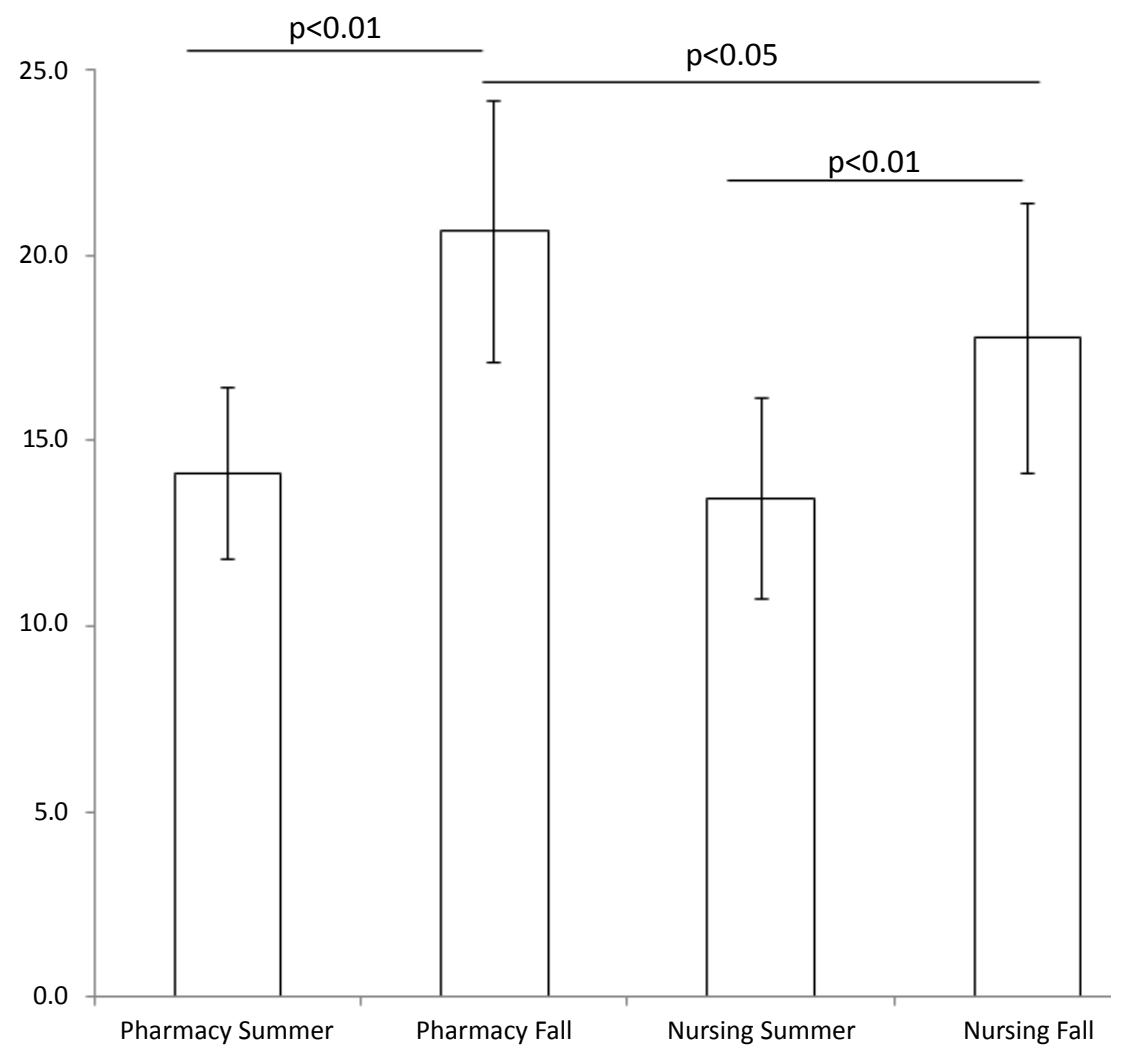

Figure 3: Perceived stress levels in pharmacy and nursing students (Mean $\pm 95 \%$ Confidence Intervals). Stress levels were determined using a 10-question Perceived Stress Scale (PSS-10) administered at the end of both Summer break and Fall term.

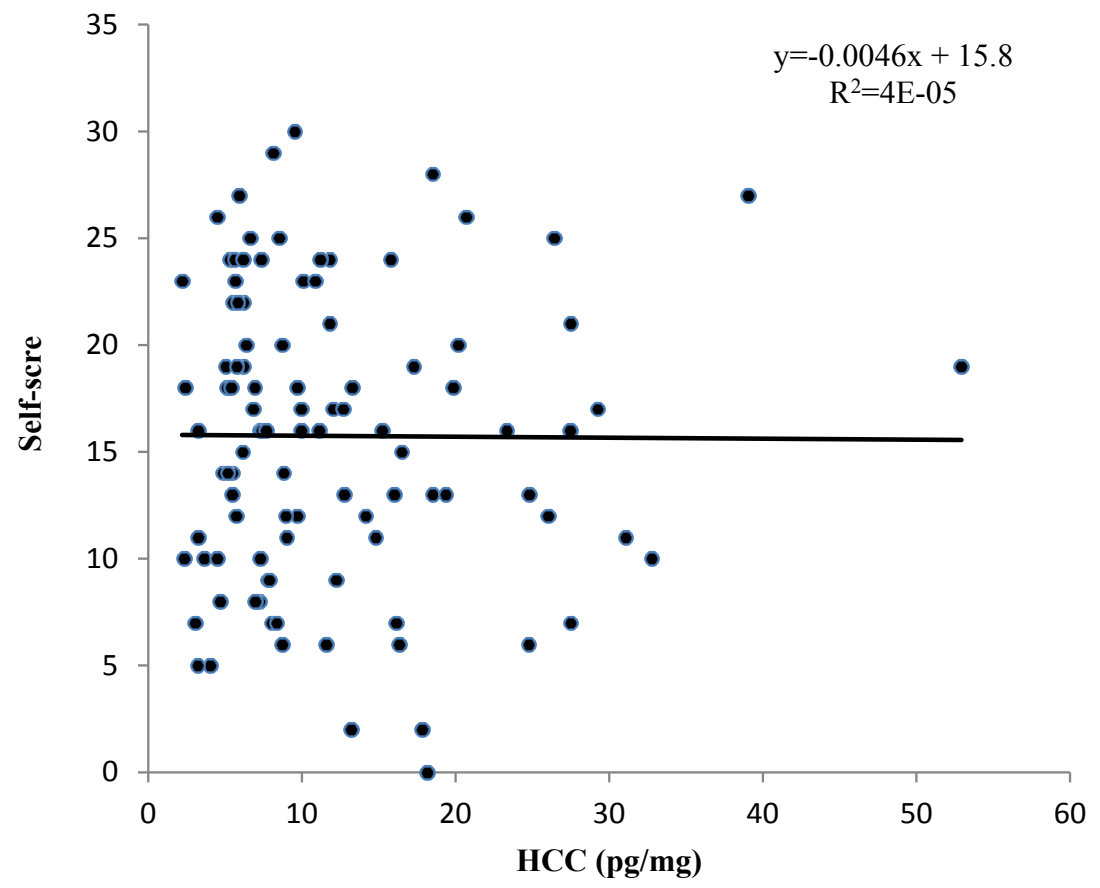

Figure 4: Linear regression analysis of perceived stress levels vs. hair cortisol concentrations (HCC). 
university students showed that "major life stressors", such as death of a relative or divorce increased hair cortisol concentrations [5]. In a study with 40 German college students subjects who stated that they had experienced a "significant mental burden" had higher hair cortisol concentrations then students who did not [6]. Similar results were obtained in a study involving students entering elementary school [7], particularly in student classified as "highly fearful". In all three studies, it seems that high cortisol concentrations in hair may have been driven by unusually threatening circumstances. In contrast to these studies, the level of stress in the present study may not have been of sufficient intensity to trigger a cortisol response. A recent study by Nejed et al. [4] examined cortisol concentrations in facial hair and nails of university students. The authors found that cortisol level was higher during a 10 day period of exams compared to a 10 day exam-free period. These results are at odds with the results of the present study. One possibility for the difference is that the facial hair collected over 10 days experiences less "wash out" (described below) than scalp hair collected for 3 months. Another possibility is that stress levels experienced during a period of continuous examinations are greater than those experienced (on average) over an entire semester.

Limitations to the present study include the inherent subjective nature of the survey instrument used to determine perceived stress and the technical challenges associated with measuring hair-cortisol concentrations. The average hair cortisol concentration found in the present study $(11 \mathrm{pg} / \mathrm{mg})$ is similar to the normal concentration (13 $\mathrm{pg} / \mathrm{mg}$ ) reported for human hair (Rocky Mountain analytical services, Calgary, Alberta, Canada) suggesting that the bioanalytical results reported here are valid. However, hair cortisol is known to be influenced by a number of factors that are difficult to control and contribute to what might be called "wash out". These include exposure to sun, hair texture and composition, hair bleaching and dye treatment, and hair washing $[9,20]$. All of these factors contribute to the variability of the hair-cortisol results and it is possible that including a larger number of students in our study or better controlling some of the variables described above would have revealed differences in hair-cortisol concentrations between the groups.

The results of the present study suggest that nursing and pharmacy students at PBA experience stress but not to the extent that it triggers the release of cortisol over the long term ( 3 months). To the best of our knowledge, this is the first study in which an objective endpoint, namely hair cortisol concentrations, has been used to asses long-term stress in pharmacy and nursing students. This end-point brings added value to the more-traditional approach of survey evaluation because it represents a quantitative marker of stress that can be compared among educational programs.

\section{References}

1. Votta RJ, Benau EM (2014) Sources of stress for pharmacy students in a nationwide sample. Curr Pharm Teach Learn 6: 675-681.
2. Gallagher CT, Mehta ANV, Selvan R, Mirza IB, Radia P, et al. (2014) Perceived stress levels among undergraduate pharmacy students in the UK. Curr Pharm Teach Learn 6: 437-441.

3. Awé C, Gaither CA, Crawford SY, Tieman J (2016) A comparative analysis of perceptions of pharmacy students' stress and stressors across two multicampus universities. Am J Pharm Educ 80: 82.

4. Nejad JG, Ghaseminezhad M, Sung K, Hoseinzadeh F, Cabibi JBA, et al. (2016) A cortisol study; facial hair and nails. J Steroids Horm 7: 2-5.

5. Karlén J, Ludvigsson J, Frostell A, Theodorsson E, Faresjö T (2011) Cortisol in hair measured in young adults - a biomarker of major life stressors? BMC Clin Pathol 11:12.

6. Ullmann E, Barthel A, Petrowski K, Stalder T, Kirschbaum C, et al. (2016) Pilot study of adrenal steroid hormones in hair as an indicator of chronic mental and physical stress. Sci Rep 6: 25842

7. Groeneveld MG, Vermeer HJ, Linting M, Noppe G, van Rossum EF, et al (2013) Children's hair cortisol as a biomarker of stress at school entry. Stress 16: $711-715$

8. Cohen S, Kamarck T, Mermelstein R (1983) A global measure of perceived stress. J Health Soc Behav 24: 385-396.

9. Russell E, Koren G, Rieder M, Van Uum S (2012) Hair cortisol as a biological marker of chronic stress: Current status, future directions and unanswered questions. Psychoneuroendocrinology 37: 589-601.

10. Wester VL, van Rossum EF (2015) Clinical applications of cortiso measurements in hair. Eur J Endocrinol 173: M1-M10.

11. Hellhammer DH, Wüst S, Kudielka BM (2009) Salivary cortisol as a biomarker in stress research. Psychoneuroendocrinology 34:163-171.

12. McEwen BS (2014) Biomarkers for assessing population and individual health and disease related to stress and adaptation. Metabolism 64: S2-S10.

13. Goldstein DS, McEwen B (2002) Allostasis, homeostats, and the nature of stress. Stress 5: 55-58.

14. Sapolsky RM, Krey LC, McEwen BS (1986) The neuroendocrinology of stress and aging: the glucocorticoid cascade hypothesis. Endocr Rev 7: 284-301.

15. Sauvé B, Koren G, Walsh G, Tokmakejian S, Van Uum SH (2007) Measurement of cortisol in human hair as a biomarker of systemic exposure. Clin Investig Med 30: 183-192.

16. Gow R, Thomson S, Rieder M, Van Uum S, Koren G (2010) An assessment of cortisol analysis in hair and its clinical applications. Forensic Sci Int 196: 32-37.

17. Chen Z, Li J, Zhang J, Xing X, Gao W, et al. (2013) Simultaneous determination of hair cortisol, cortisone and DHEAS with liquid chromatography-electrospray ionization-tandem mass spectrometry in negative mode. J Chromatogr B Anal Technol Biomed Life Sci 929: 187-194.

18. Hirsch JD, Do AH, Hollenbach KA, Manoguerra AS, Adler DS (2009) Students' health-related quality of life across the preclinical pharmacy curriculum. Am J Pharm Educ 73:1-6.

19. Marshall LL, Allison A, Nykamp D, Lanke S (2008) Perceived stress and quality of life among doctor of pharmacy students. Am J Pharm Educ 72:14-16.

20. Li J, Xie Q, Gao W, Xu Y, Wang S, et al. (2012) Time course of cortisol loss in hair segments under immersion in hot water. Clin Chim Acta 413: 434-440. 